\title{
Networked Output Feedback Control for a Class of Uncertain Nonlinear Time-Delay Systems
}

\author{
Hongyan Chu $\mathbb{D}^{1},{ }^{1}$ Yan Wang $\mathbb{D}^{2},{ }^{2}$ and Weiling $\mathrm{Li}^{3}$ \\ ${ }^{1}$ School of Physics and Technology, Nanjing Normal University, Nanjing 210096, China \\ ${ }^{2}$ School of NARI Electrical and Automation Engineering, Nanjing Normal University, Nanjing 210096, China \\ ${ }^{3}$ School of Energy and Mechanical Engineering, Nanjing Normal University, Nanjing 210096, China \\ Correspondence should be addressed to Hongyan Chu; njnuchuhongyan@163.com
}

Received 16 May 2019; Accepted 24 July 2019; Published 20 December 2019

Academic Editor: Luca Pancioni

Copyright (c) 2019 Hongyan Chu et al. This is an open access article distributed under the Creative Commons Attribution License, which permits unrestricted use, distribution, and reproduction in any medium, provided the original work is properly cited.

\begin{abstract}
This paper studies the global stabilization problem for a class of uncertain time-delay nonlinear systems by designing a sampleddata output feedback controller via network. Under a lower triangular linear growth condition, when only the output is measurable, a sampled-data output feedback network controller, whose observer and control law are both linear, is constructed to solve the stabilization problem. Using a feedback domination design approach which substantially differs from the separation principle, we explicitly construct a Lyapunov-Krasovskill functional to prove the global asymptotic stability with the help of inductive proof method. The control law is discrete-time and linear, hence simulation examples be easily implemented with computers to show the effectiveness of our proposed method.
\end{abstract}

\section{Introduction and Overview}

In this paper, we will extend the results in [1] to the nonlinear networked systems when the control input is transmitted through the shared common-use network. A more sophisticated estimation technique is introduced to estimate the state growth in the presence of network delay. The uncertain time-delay nonlinear system with input delay is described as follows:

$$
\begin{aligned}
\dot{x}_{1}(t) & =x_{2}(t)+\bar{\phi}_{1}(t, x(t), x(t-\tau)), \\
\vdots & \\
\dot{x}_{n-1}(t) & =x_{n}(t)+\bar{\phi}_{n-1}(t, x(t), x(t-\tau)), \\
\dot{x}_{n}(t) & =u(t-\tau)+\bar{\phi}_{n}(t, x(t), x(t-\tau)), \\
y(t) & =x_{1}(t), \\
x(t) & =\psi(t), \quad-\tau \leq t \leq 0,
\end{aligned}
$$

where $x(t)=\left(x_{1}(t), \ldots, x_{n}(t)\right)^{T} \in R^{n}$ is the system state, $u(t) \in R$ is the control input which is transmitted through the shared network. For $i=1, \ldots, n, \bar{\phi}_{i}(\cdot)$ is an unknown nonlinear function of the states in lower triangular form, which might be neither known precisely nor vanishing at the origin. $\tau \geq 0$ is time delay which can be represented by $N T$, where $T$ is the sampling period. $\psi(t)$ is the initial function of the system state vector. The structure of the system is illustrated in Figure 1. The control objective is to design a sampled-data output feedback networked controller to globally stabilize the nonlinear system (1).

Nowadays, we can almost do nothing without computers with the widespread application of computer technology, especially in engineering systems and control systems. The computer controlled systems are also referred to as sampleddata control systems to emphasize the sampling process as their crucial feature. As a result, design of sampling-data controller is more imperative and practical in real world [2]. For a linear system, a sampled-data linear continuous-time system is equivalent to a linear discrete-time system $[3,4]$ due to the separation principle [5]. However, this convenience is unfortunately invalid when a nonlinear system is considered.

When all the nonlinear functions are known precisely, the problem of stabilization can be easily solved using state feedback sampled-data controller $[6,7]$ and the references therein.

In the case when full-states are not available, the global output feedback stabilization problem has to be addressed by using a sampled-data output feedback controller. In the literature, the sampled-data output feedback stabilization problem 


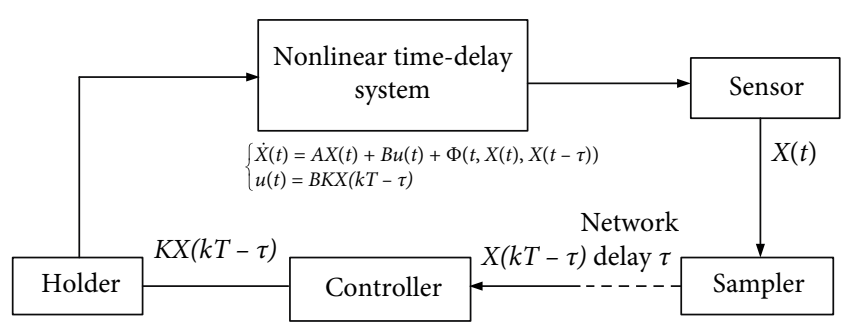

Figure 1: Structure of the nonlinear network system via sampleddata controller.

for a class of nonlinear systems has been investigated by designing discrete-time high-gain observers in [8-12] to semiglobal or local stabilize the nonlinear systems by choosing a small enough sampling period and sufficiently large observer gain. Du et al. [13] discussed the global sampled-data output feedback stabilization problem for the nonlinear system under a homogeneous growth condition. In [14], under the same lower-triangular linear growth condition for the unknown nonlinearities as in [15], a sampled-data output feedback controller is explicitly constructed to globally stabilize the system (1) for the case of $\tau=0$. However, for the case of $\tau>0$, which extensively exists in many practical systems such as communication-based systems, biological systems, multiagent systems, and so on, some essential difficulties will inevitably be encountered in constructing the desired controllers.

In the literature, there are a few results for the nonlinear time-delay systems without controllable linearization. In [16], by extending the adding a power integrator technique to the stochastic time delay systems, a state-feedback controller is explicitly constructed to globally stabilize a class of lower triangular stochastic time-delay nonlinear systems in probability. In $[17,18]$, by using the methods of adding a power integrator and backstepping, a continuous state-feedback controller is constructed to globally stabilize a class of high-order nonlinear time-delay systems. In [19], both state feedback and output feedback controllers are investigated for a class of feed-forward nonlinear time-delay systems by designing a dynamic equation. In [20], a universal-type adaptive output feedback controller with unknown growth rate is designed to solve the adaptive control problem for a class of uncertain nonlinear time delay systems with unknown output functions. In [1], a sampled-data output feedback controller is designed for nonlinear systems with time-delays in states, a new estimation technique and an inductive method were introduced firstly to estimate the state growth in the presence of time delay and nonvarnishing nonlinearities.

All the achievements above are received with the assumption that the control signal are transmitted without delay, when the system output is sampled and transmitted through a shared band-limited network, the problem of output feedback stabilization for system (1) is known as more challenging. Some results are got with a constraint condition that the maximum delay must be less than the minimum sampling period [21-24]. Du et al. [25] discussed the global sampled-data output feedback stabilization problem for the upper-triangular nonlinear systems with input delay. In [26], a class of lower triangular systems with time delays both in the nonlinearities and control input are discussed, while the input delay is time-varying but bounded. This paper will show that under the same assumption used in [20,27], i.e., the lower triangular linear growth condition for the unknown nonlinearities with time-delay, we can explicitly construct a sampled-data output feedback network controller to globally stabilize system (1). Because of the existence of time delays and input delays, the estimation schemes in $[1,14]$ will be no longer applicable and a more subtle estimation method is needed to identify the maximum allowable sampling period.

The paper is organized as follows: in Section 2, the problem is formulated and the assumptions are presented. The main results of the paper is contained in Section 3, where an inductive method is introduced to estimate the state growth within one sampling period. Finally, an example in Section 5 is illustrated and some concluding remarks are given in the Acknowledgments respectively.

\section{Problem Statement and Assumptions}

Our objective is to find, if possible, a linear sampled-data output feedback control which is transmitted through network

$$
\begin{aligned}
\eta\left(t_{k+1}\right) & =F\left(\eta\left(t_{k}\right), y\left(t_{k}\right)\right) \\
u(t) & =u\left(t_{k}-\tau\right), \quad \forall t \in\left[t_{k}, t_{k+1}\right), \quad t_{k}=k T, \quad k=0,1,2, \ldots,
\end{aligned}
$$

such that the sampled-data controller globally stabilizes the nonlinear time-delay system (1) and (2).

In order to obtain our objective, we make the following three assumptions.

Assumption 1. There exist constants $c_{1}>0, c_{2}>0$ such that for $i=1, \ldots, n$, the following holds

$$
\begin{aligned}
& \left|\bar{\phi}_{i}(t, x(t), x(t-\tau))\right| \leq c_{1}\left(\left|x_{1}(t)\right|+\left|x_{2}(t)\right|+\cdots+\left|x_{i}(t)\right|\right) \\
& +c_{2}\left(\left|x_{1}(t-\tau)\right|+\left|x_{2}(t-\tau)\right|+\cdots+\left|x_{i}(t-\tau)\right|\right) .
\end{aligned}
$$

Remark 2. When $\tau=0$, Assumption 1 is transformed into the linear growth condition in $[14,15]$. It has been shown in [15], based on a domination approach, the global stabilization problem is solvable using linear output feedback. Later, in [14], the global asymptotic stabilization is also solvable using a sample-data output feedback controller with an appropriate sampling period. The objective of this paper is to prove that when $\tau$ is not zero, there exists a sampled-data controller which globally asymptotically stabilizes the uncertain nonlinear system (1) and (2).

Assumption 3. For the simplicity of analysis and proof, we assume that time delay $\tau$ equals to sampling period $T$.

Remark 4. We make the assumption only to make it easy to deal with. But it is not necessary that the time delay has to be same with $T$. When $\tau$ less than $T$, for the sampled-data system, it equals to $\tau=T$. While when $\tau$ is bigger than $T$, it can also be transacted using our technology as well but a little complex. On the other hand, for the networked control system, when the delay is too big, we sometimes looked on the signal as a packet dropout, and replace it with the previous signal. As a result, it 
is more meaningful to make a research on the one-sample-time delay events.

Assumption 5. We assume that the initial states satisfy

$$
-\frac{7}{3}\|x(0)\| \leq \psi(t) \leq \frac{7}{3}\|x(0)\|, \quad \forall t \in[-\tau, 0) .
$$

Remark 6. For most of the delay systems, the initial states will stay within a certain range of the states of time 0 because of continuity. As a result, the Assumption 5 is reasonable in real systems.

\section{Output-Feedback Networked Control}

In this section, we show that the problem of output feedback stabilization for system (1) and (2) under sampled-data control is solvable under Assumptions 1, 3, and 5.

Theorem 7. Under Assumptions 1, 3, and 5, there exists an appropriate sampling period $T$ such that the linear sampleddata output feedback controller (2) globally asymptotically stabilizes the nonlinear time-delay system (1).

Proof. The design of the discrete-time controller includes four parts. At the first beginning, we introduce a gain $L$ which can be scaled so as to dominate the nonlinear item. Next, assuming that not all the states are measurable, we construct a linear discrete-time observer according to the output. Then we construct a Lyapunov-Krasovskii function and pay specially attention to the transaction of the item $\left\|\eta(t)-\eta\left(t_{k}\right)\right\|$. The scaling gain $L$ is also given in this section in order to make the choice of $T$ easy. Finally, it is shown that there exists an appropriate sampling period $T$ to globally asymptotically stabilize the closed-loop system.

3.1.Pre-Treatment of the System (1): Change of Coordinates. First we introduce the following change of coordinates with a scaling gain $L>1$ to be determined later

$$
\begin{aligned}
z_{i}(t) & =\frac{x_{i}(t)}{L^{i-1}}, \\
z_{i}(t-\tau) & =\frac{x_{i}(t-\tau)}{L^{i-1}}, \quad i=1, \ldots, n, \\
v(t) & =\frac{u(t)}{L^{n}} .
\end{aligned}
$$

Under (5), system (1) can be rewritten as

$$
\begin{aligned}
\dot{z}_{1}(t) & =L z_{2}(t)+\phi_{1}(t, z(t), z(t-\tau)), \\
\vdots & \\
\dot{z}_{n-1}(t) & =L z_{n}(t)+\phi_{n-1}(t, z(t), z(t-\tau)), \\
\dot{z}_{n}(t) & =L v(t)+\phi_{n}(t, z(t), z(t-\tau)), \\
y(t) & =z_{1}(t), \\
z(t) & =\phi(t), \quad-\tau \leq t \leq 0,
\end{aligned}
$$

where $\phi_{i}(t, z(t), z(t-\tau))=\bar{\phi}_{i}(t, x(t), x(t-\tau)) / L^{i-1}$, for $i=1, \ldots, n$.

$$
\begin{aligned}
\left|\phi_{i}(t, z(t), z(t-\tau))\right|= & \frac{\left|\bar{\phi}_{i}(t, x(t), x(t-\tau))\right|}{L^{i-1}} \\
\leq & \frac{1}{L^{i-1}}\left[c_{1}\left(\left|x_{1}(t)\right|+\cdots+\left|x_{i}(t)\right|\right)\right. \\
& \left.+c_{2}\left(\left|x_{1}(t-\tau)\right|+\cdots+\left|x_{i}(t-\tau)\right|\right)\right] \\
= & \frac{1}{L^{i-1}}\left[c_{1}\left(\left|z_{1}(t)\right|+\cdots+L^{i-1}\left|z_{i}(t)\right|\right)\right. \\
& \left.+c_{2}\left(\left|z_{1}(t-\tau)\right|+\cdots+L^{i-1}\left|z_{i}(t-\tau)\right|\right)\right] \\
\leq & {\left[c_{1}\left(\left|z_{1}(t)\right|+\cdots+\left|z_{i}(t)\right|\right)\right.} \\
& \left.+c_{2}\left(\left|z_{1}(t-\tau)\right|+\cdots+\left|z_{i}(t-\tau)\right|\right)\right] .
\end{aligned}
$$

According to (7), the new nonlinearity item will satisfy the following Assumption 8.

Assumption 8. There exist constants $c_{1}>0, c_{2}>0$ such that

$$
\begin{aligned}
& \left|\phi_{i}(t, z(t), z(t-\tau))\right| \leq c_{1}\left(\left|z_{1}(t)\right|+\cdots+\left|z_{i}(t)\right|\right) \\
& \quad+c_{2}\left(\left|z_{1}(t-\tau)\right|+\cdots+\left|z_{i}(t-\tau)\right|\right) .
\end{aligned}
$$

Defining

$$
\begin{aligned}
& z(t)=\left[\begin{array}{c}
z_{1}(t) \\
\vdots \\
z_{n-1}(t) \\
z_{n}(t)
\end{array}\right], \\
& \Phi(\cdot)=\left[\begin{array}{c}
\phi_{1}(\cdot) \\
\vdots \\
\phi_{n-1}(\cdot) \\
\phi_{n}(\cdot)
\end{array}\right] \text {, } \\
& A=\left[\begin{array}{cccc}
0 & 1 & \ldots & 0 \\
\vdots & \vdots & \ddots & \vdots \\
0 & 0 & \ldots & 1 \\
0 & 0 & \ldots & 0
\end{array}\right] \text {, } \\
& B=\left[\begin{array}{c}
0 \\
\vdots \\
0 \\
1
\end{array}\right] \text {, } \\
& C=\left[\begin{array}{c}
1 \\
\vdots \\
0 \\
0
\end{array}\right]^{T} \text {, }
\end{aligned}
$$

where $(\cdot)=(t, z(t), z(t-\tau))$.

With the help of the aforementioned definition, the closedloop system (6) is transformed into

$$
\begin{aligned}
& \dot{z}(t)=L A z(t)+L B v(t)+\Phi(\cdot), \\
& y(t)=C z(t) .
\end{aligned}
$$

Since only $y(t)$ is measurable at sampling point, we will design a discrete-time observer to estimate the unmeasurable states.

3.2. The Augmented System Combing a Linear Discrete-Time Observer with the Original System (10). Using the method in [14], we design the following discrete-time observer over $\left[t_{k}, t_{k+1}\right)$ 


$$
\begin{aligned}
\hat{z}_{1}(t) & =L \hat{z}_{2}(t)+L a_{1}\left(z_{1}\left(t_{k}\right)-\hat{z}_{1}(t)\right), \\
\vdots & \\
\hat{z}_{n-1}(t) & =L \hat{z}_{n}(t)+L a_{n-1}\left(z_{1}\left(t_{k}\right)-\hat{z}_{1}(t)\right), \\
\hat{z}_{n}(t) & =L v\left(t_{k}\right)+L a_{n}\left(z_{1}\left(t_{k}\right)-\hat{z}_{1}(t)\right),
\end{aligned}
$$

where $a_{j}, j=1, \ldots, n$ are coefficients of the Hurwitz polyno$\operatorname{mial} p(s)=s^{n}+a_{n} s^{n-1}+\cdots+a_{2} s+a_{1}$.

With the help of the following notations

$$
\begin{aligned}
\hat{z}(t) & =\left[\begin{array}{llll}
\hat{z}_{1}(t) & \ldots & \hat{z}_{n-1}(t) & \hat{z}_{n}(t)
\end{array}\right]^{T}, \\
H & =\left[\begin{array}{llll}
a_{1} & \ldots & a_{n-1} & a_{n}
\end{array}\right]^{T}, \\
\hat{A} & =A-H C,
\end{aligned}
$$

(11) is rewritten as

$$
\dot{\hat{z}}(t)=L \hat{A} \hat{z}(t)+L B v\left(t_{k}\right)+L H C z\left(t_{k}\right) .
$$

We design the output feedback sampled-data controller as

$$
v(t)=v\left(t_{k}\right)=-K \hat{z}\left(t_{k}-\tau\right)=-K \hat{z}\left(t_{k-1}\right), \quad \forall t \in\left[t_{k}, t_{k+1}\right) .
$$

Applying the output feedback controller (14) into the system (10) and (13), the new augmented system can be described as

$$
\begin{aligned}
\dot{Z}(t)= & {\left[\begin{array}{c}
\dot{z}(t) \\
\dot{\tilde{z}}(t)
\end{array}\right]=L\left[\begin{array}{cc}
A & 0 \\
0 & \hat{A}
\end{array}\right] Z(t)-L\left[\begin{array}{cc}
0 & B K \\
0 & B K
\end{array}\right] Z\left(t_{k-1}\right) } \\
& +L\left[\begin{array}{cc}
0 & 0 \\
H C & 0
\end{array}\right] Z\left(t_{k}\right)+\left[\begin{array}{c}
\Phi(\cdot) \\
0
\end{array}\right],
\end{aligned}
$$

$\left\|Z(t)-Z\left(t_{k-1}\right)\right\|$ can be separated as the following two items: $\left\|Z(t)-Z\left(t_{k-1}\right)\right\| \leq\left\|Z(t)-Z\left(t_{k}\right)\right\|+\left\|Z\left(t_{k}\right)-Z\left(t_{k-1}\right)\right\|$.

Separating $\left(Z(t)-Z\left(t_{k-1}\right)\right)$ into two items, the augmented system can be transformed into:

$$
\begin{aligned}
\dot{Z}(t)= & L\left[\begin{array}{cc}
A & -B K \\
H C & \hat{A}-B K
\end{array}\right] Z(t)+L\left[\begin{array}{cc}
0 & B K \\
0 & B K
\end{array}\right]\left(Z(t)-Z\left(t_{k-1}\right)\right) \\
& +L\left[\begin{array}{cc}
0 & 0 \\
-H C & 0
\end{array}\right]\left(Z(t)-Z\left(t_{k}\right)\right)+\left[\begin{array}{c}
\Phi(\cdot) \\
0
\end{array}\right] \\
= & L\left[\begin{array}{cc}
A & -B K \\
H C & \hat{A}-B K
\end{array}\right] Z(t)+L\left[\begin{array}{cc}
0 & B K \\
-H C & B K
\end{array}\right]\left(Z(t)-Z\left(t_{k}\right)\right) \\
& +L\left[\begin{array}{cc}
0 & B K \\
0 & B K
\end{array}\right]\left(Z\left(t_{k}\right)-Z\left(t_{k-1}\right)\right)+\left[\begin{array}{c}
\Phi(\cdot) \\
0
\end{array}\right] .
\end{aligned}
$$

Denote

$$
\mathscr{A}=\left[\begin{array}{cc}
A & -B K \\
H C & \hat{A}-B K
\end{array}\right]
$$

which can be represented by

$$
\mathscr{A}=\left[\begin{array}{cc}
0 & I \\
-I & I
\end{array}\right]\left[\begin{array}{cc}
\hat{A} & 0 \\
B K & A-B K
\end{array}\right]\left[\begin{array}{cc}
I & -I \\
I & 0
\end{array}\right] .
$$

This with the fact that $\hat{A}$ and $A-B K$ are Hurwitz matrices, implies that $\mathscr{A}$ is a Hurwitz matrix as well. Therefore, there exists a positive matrix $P=P^{T}$ such that $\mathscr{A}^{T} P+P \mathscr{A}=-I$.

3.3. Construction of Lyapunov-Krasovskii Functional as well as the Transaction of the Derivative. In this section, we first construct a candidate Lyapunov-Krasovskii functional as follows

$$
V(t, Z(t), Z(t-\tau))=Z^{T}(t) P Z(t)+\int_{t-\tau}^{t} Z^{2}(s) d s .
$$

Noting that $\|B\|=1$ and $\mathscr{A}$ is a Hurwitz matrix, then the derivative of $V(t, Z(t), Z(t-\tau))$ along the system (15) is $\dot{V}(t, Z(t), Z(t-\tau))=2 Z^{T}(t) P \dot{Z}(t)+Z(t)^{2}-Z(t-\tau)^{2}$

$$
\begin{aligned}
& \quad \leq-L\|Z(t)\|^{2}+2 L\|Z(t)\|\|P\|(\|K\| \\
& +\|H\|)\left\|Z(t)-Z\left(t_{k}\right)\right\| \\
& +2 L\|K\|\|Z(t)\|\left(\left\|Z\left(t_{k}\right)-Z\left(t_{k-1}\right)\right\|\right) \\
& +2\|Z(t)\|\|P\|\|\Phi(\cdot)\|+\|Z(t)\|^{2}-\|Z(t-\tau)\|^{2} .
\end{aligned}
$$

The nonlinear item $\Phi(\cdot)$ satisfies

$$
\begin{aligned}
\|\Phi(\cdot)\| \leq & \sqrt{\left|\phi_{1}(t)\right|^{2}+\left|\phi_{2}(t)\right|^{2}+\cdots+\left|\phi_{n}(t)\right|^{2}} \\
= & \sqrt{\left[c_{1}\left|z_{1}(t)\right|+c_{2}\left|z_{1}(t-\tau)\right|\right]^{2}+\left[c_{1}\left(\left|z_{1}(t)\right|+\left|z_{2}(t)\right|\right)+c_{2}\left(\left|z_{1}(t-\tau)\right|+\left|z_{2}(t-\tau)\right|\right)\right]^{2}} \cdots \\
& \sqrt{+\cdots+\left[c_{1}\left(\left|z_{1}(t)+\cdots+\right| z_{n}(t) \mid\right)+c_{2}\left(\left|z_{1}(t-\tau)+\cdots+\right| z_{n}(t-\tau) \mid\right)\right]^{2}} \\
\leq & \sqrt{(2+4+\cdots+2 n) c_{1}^{2}\|Z(t)\|^{2}+((2+4+\cdots+2 n)) c_{2}^{2}\|Z(t-\tau)\|^{2}} \\
\leq & c_{1} c_{3}\|Z(t)\|+c_{2} c_{3}\|Z(t-\tau)\|,
\end{aligned}
$$

where $c_{3}=\sqrt{n(n+1)}$.

Substituting (22) into (21), we can get that $\dot{V}(t, Z(t), Z(t-\tau)) \leq-L\|Z(t)\|^{2}+2 L\|Z(t)\|\|P\|(\|K\|+\|H\|)\left\|Z(t)-Z\left(t_{k}\right)\right\|$ $+2 L\|K\|\|Z(t)\|\left(\left\|Z\left(t_{k}\right)-Z\left(t_{k-1}\right)\right\|\right)+2 c_{1} c_{3}\|P\|\|Z(t)\|^{2}$

$+c_{2}^{2} c_{3}^{2}\|P\|^{2}\|Z(t)\|^{2}+\|Z(t-\tau)\|^{2}+\|Z(t)\|^{2}-\|Z(t-\tau)\|^{2}$

$=-\left(L-2 c_{1} c_{3}\|P\|-c_{2}^{2} c_{3}^{2}\|P\|^{2}-1\right)\|Z(t)\|^{2}$

$+2 L\|P\|(\|K\|+\|H\|)\|Z(t)\|\left\|Z(t)-Z\left(t_{k}\right)\right\|$

$+2 L\|P\|\|K\|\|Z(t)\|\left\|Z\left(t_{k}\right)-Z\left(t_{k-1}\right)\right\|$.

Selecting

$$
L=3+2 c_{1} c_{3}\|P\|+c_{2}^{2} c_{3}^{2}\|P\|^{2}
$$

then (23) can be changed into

$$
\begin{gathered}
\dot{V}(t, Z(t), Z(t-\tau)) \leq-2\|Z(t)\|^{2}+2 L\|P\|(\|K\|+\|H\|)\|Z(t)\| \\
\cdot\left\|Z(t)-Z\left(t_{k}\right)\right\|+2 L\|P\|\|K\|\|Z(t)\|\left\|Z\left(t_{k}\right)-Z\left(t_{k-1}\right)\right\| .
\end{gathered}
$$

In the following, we will discuss how to use the inductive method to deal with the item $\left\|Z(t)-Z\left(t_{k}\right)\right\|$ and $\left\|Z\left(t_{k}\right)-Z\left(t_{k-1}\right)\right\|$.

$$
\begin{aligned}
\left\|Z(t)-Z\left(t_{k}\right)\right\| \leq & \int_{t_{k}}^{t}\|\dot{Z}(s)\| d s \\
\leq & \int_{t_{k}}^{t}\left[L\|\bar{A}\|+c_{1} c_{3}\right]\|Z(s)\|+c_{2} c_{3}\|Z(s-\tau)\| \\
& \left.+L\|H\| Z\left(t_{k}\right)\right]+L\|K\|\left\|Z\left(t_{k-1}\right)\right\| d s \\
= & \int_{t_{k}}^{t}\left[d_{1}\|Z(s)\|+d_{2}\left\|Z\left(t_{k}\right)\right\|+d_{3}\left\|Z\left(t_{k-1}\right)\right\|\right] d s \\
& +\int_{t_{k}}^{t} d_{4}\|Z(s-\tau)\| d s \\
= & A(t)+\int_{t_{k}}^{t} d_{4}\|Z(s-\tau)\| d s,
\end{aligned}
$$


where

$$
\begin{aligned}
\bar{A} & =\left[\begin{array}{cc}
A & 0 \\
0 & \hat{A}
\end{array}\right], \\
d_{1} & =L\|\bar{A}\|+c_{1} c_{3}, \\
d_{2} & =L\|H\|, \\
d_{3} & =L\|K\|, \\
d_{4} & =c_{2} c_{3}, \\
A(t)=\int_{t_{k}}^{t}\left[d_{1}\|z(s)\|\right. & \left.+d_{2}\left\|Z\left(t_{k}\right)\right\|+d_{3}\left\|z\left(t_{k-1}\right)\right\|\right] d s .
\end{aligned}
$$

In the case of $\tau=T$, (29) can be illustrated as

$$
\left\|Z(t)-Z\left(t_{k}\right)\right\| \leq A(t)+\int_{t_{k-1}}^{t_{k}} d_{4}\|Z(s)\| d s .
$$

The derivative of $A(t)$ can be written as

$$
\begin{aligned}
\dot{A}(t)= & d_{1}\|Z(t)\|+d_{2}\left\|Z\left(t_{k}\right)\right\|+d_{3}\left\|Z\left(t_{k-1}\right)\right\| \\
\leq & d_{1}\left\|Z(t)-Z\left(t_{k}\right)\right\|+\left(d_{1}+d_{2}\right)\left\|Z\left(t_{k}\right)\right\|+d_{3}\left\|Z\left(t_{k-1}\right)\right\| \\
\leq & d_{1} A(t)+d_{1} d_{3} \int_{t_{k-1}}^{t_{k}}\|Z(s)\| d s \\
& +\left(d_{1}+d_{2}\right)\left\|Z\left(t_{k}\right)\right\|+d_{3}\left\|Z\left(t_{k-1}\right)\right\| .
\end{aligned}
$$

The solution of $A(t)$ is

$$
\begin{aligned}
A(t) \leq & e^{d_{1}\left(t-t_{k}\right)} \int_{t_{k}}^{t} e^{-d_{1}\left(s-t_{k}\right)}\left[d_{1} d_{3} \int_{t_{k-1}}^{t_{k}}\|Z(a)\| d a\right. \\
& \left.+\left(d_{1}+d_{2}\right)\left\|Z\left(t_{k}\right)\right\|+d_{3}\left\|Z\left(t_{k-1}\right)\right\|\right] d s \\
\leq & d_{3}\left[e^{d_{1} T}-1\right] \int_{t_{k-1}}^{t_{k}}\|Z(a)\| d a+\left[e^{d_{1} T}-1\right] \\
& \cdot \frac{\left(d_{1}+d_{2}\right)\left\|Z\left(t_{k}\right)\right\|+d_{3}\left\|Z\left(t_{k-1}\right)\right\|}{d_{1}} .
\end{aligned}
$$

As a result

$$
\begin{aligned}
\left\|Z(t)-Z\left(t_{k}\right)\right\| \leq & d_{3} e^{d_{1} T} \int_{t_{k-1}}^{t_{k}}\|Z(s)\| d s \\
& +\left[e^{d_{1} T}-1\right] \frac{\left(d_{1}+d_{2}\right)\left\|Z\left(t_{k}\right)\right\|+d_{3}\left\|Z\left(t_{k-1}\right)\right\|}{d_{1}} .
\end{aligned}
$$

Step 1. When $t_{k}=0, t \in[0, T)$.

$$
\begin{aligned}
\|Z(t)-Z(0)\| \leq & d_{3} e^{d_{1} T} \int_{-T}^{0}\|Z(s)\| d s \\
& +\left[e^{d_{1} T}-1\right] \frac{\left(d_{1}+d_{2}\right)\|Z(0)\|+d_{3}\|Z(-T)\|}{d_{1}} .
\end{aligned}
$$

According to Assumption 5, we can get that

$\|Z(t)-Z(0)\| \leq\left(\frac{7}{3} d_{3} e^{d_{1} T} T+\left[e^{d_{1} T}-1\right] \frac{d_{1}+d_{2}+7 / 3 d_{3}}{d_{1}}\right)\|Z(0)\|$.

Define

$$
\frac{7}{3} d_{3} e^{d_{1} T} T+\frac{\left(d_{1}+d_{2}\right)+7 / 3 d_{3}}{d_{1}}\left[e^{d_{1} T}-1\right] \leq \delta(T),
$$

where $\delta(T)$ is a variable relating to $T$ and it satisfies $0<\delta(T)<2 / 5$.

With the help of (35), it can be concluded that

$$
\begin{aligned}
& \|Z(t)-Z(0)\| \leq \delta(T)\|Z(0)\| \\
& \quad(1-\delta(T))\|Z(0)\| \leq\|Z(t)\| \leq(1+\delta(T))\|Z(0)\| .
\end{aligned}
$$

Because the system is continuous, we can get that

$$
(1-\delta(T))\|Z(0)\| \leq\|Z(T)\| \leq(1+\delta(T))\|Z(0)\| .
$$

Step 2. When $t_{k}=T, t \in[T, 2 T)$.

$$
\begin{aligned}
\|Z(t)-Z(T)\| \leq & d_{3} e^{d_{1} T} \int_{0}^{T}\|Z(s)\| d s+\left[e^{d_{1} T}-1\right] \\
& \cdot \frac{\left(d_{1}+d_{2}\right)\|Z(T)\|+d_{3}\|Z(0)\|}{d_{1}} \\
\leq & \frac{1+\delta(T)}{1-\delta(T)} d_{3} e^{d_{1} T} T\|Z(T)\|+\left[e^{d_{1} T}-1\right] \\
& \cdot \frac{\left(d_{1}+d_{2}\right)+d_{3} /(1-\delta(T))}{d_{1}}\|Z(T)\| \\
\leq & \frac{7}{3} d_{3} e^{d_{1} T} T\|Z(T)\|+\left[e^{d_{1} T}-1\right] \\
& \cdot \frac{\left(d_{1}+d_{2}\right)+(5 / 3) d_{3}}{d_{1}}\|Z(T)\| .
\end{aligned}
$$

With the help of (35), it can be deduced that

$$
(1-\delta(T))\|Z(T)\| \leq\|Z(t)\| \leq(1+\delta(T))\|Z(T)\| .
$$

Inductive step: supposing at step $k-1, t_{k}=(k-1) T$, $t \in[(k-1) T, k T], Z(t)$ satisfies

$(1-\delta(T))\|Z((k-1) T)\| \leq\|Z(t)\| \leq(1+\delta(T))\|Z((k-1) T)\|$.

We claim that (40) also holds at step $k, t_{k}=k T, t \in[k T,(k+1) T)$.

Proof. Substituting $t_{k}=k T$ into (32), it can be rewritten as

$$
\begin{aligned}
\|Z(t)-Z(k T)\| \leq & d_{3} e^{d_{1} T} \int_{(k-1) T}^{k T}\|Z(s)\| d s+\left[e^{d_{1} T}-1\right] \frac{\left(d_{1}+d_{2}\right)\|Z(k T)\|+d_{3}\|Z((k-1) T)\|}{d_{1}} \\
\leq & (1+\delta(T)) d_{3} e^{d_{1} T} T\|Z((k-1) T)\| \\
& +\left[e^{d_{1} T}-1\right] \frac{\left(d_{1}+d_{2}\right)\|Z(k T)\|+\left(d_{3} /(1-\delta(T))\right)\|Z(k T)\|}{d_{1}} \\
\leq & \frac{7}{3} d_{3} e^{d_{1} T} T\|Z(k T)\|+\left[e^{d_{1} T}-1\right] \frac{\left(d_{1}+d_{2}\right)+(5 / 3) d_{3}}{d_{1}}\|Z(k T)\| .
\end{aligned}
$$


With the help of (35), it can be deduced that

$$
(1-\delta(T))\|Z(k T)\| \leq\|Z(t)\| \leq(1+\delta(T))\|Z(k T)\| .
$$

From above, we can conclude that for every $t \in[k T,(k+1) T)$,

$$
\left\|Z(t)-Z\left(t_{k}\right)\right\| \leq \delta(T)\left\|Z\left(t_{k}\right)\right\| .
$$

When $t \in\left[t_{k-1}, t_{k}\right)$,

$$
\left\|Z(t)-Z\left(t_{k-1}\right)\right\| \leq \delta(T)\left\|Z\left(t_{k-1}\right)\right\| .
$$

That is to say the following holds

$$
\left\|Z\left(t_{k}\right)-Z\left(t_{k-1}\right)\right\| \leq \delta(T)\left\|Z\left(t_{k-1}\right)\right\| .
$$

Substituting (43) and (45) into (25) when $t \in\left[t_{k}, t_{k+1}\right)$

$$
\begin{aligned}
\dot{V}(t, Z(t), Z(t-\tau)) \leq & -2\|Z(t)\|^{2} \\
& +2 L\|P\|(\|K\|+\|H\|) \delta(T)\|Z(t)\|\left\|Z\left(t_{k}\right)\right\| \\
& +2 L\|P\|\|H\| \delta(T)\|Z(t)\|\left\|Z\left(t_{k-1}\right)\right\| \\
\leq & -2\|Z(t)\|^{2}+2 L\|P\|(\|K\|+\|H\|) \\
& \cdot \frac{\delta(T)}{1-\delta(T)}\|Z(t)\|^{2}+2 L\|P\|\|K\| \frac{\delta(T)}{(1-\delta(T))^{2}}\|Z(t)\|^{2} \\
\leq & -2\|Z(t)\|^{2}+2 L\|P\|\|K\| \frac{2 \delta(T)-(\delta(T))^{2}}{(1-\delta(T))^{2}}\|Z(t)\|^{2} \\
& +2 L\|P\|\|H\| \frac{\delta(T)}{1-\delta(T)}\|Z(t)\|^{2} .
\end{aligned}
$$

If we choose an appropriate $T$ such that the following two inequalities are satisfied

$$
\begin{gathered}
2-2 L\|P\|\|K\| \frac{2 \delta(T)-(\delta(T))^{2}}{(1-\delta(T))^{2}}-2 L\|P\|\|H\| \frac{\delta(T)}{1-\delta(T)}<0 \\
\frac{7}{3} d_{3} e^{d_{1} T} T+\frac{d_{1}+5 / 3 d_{2}}{d_{1}}\left[e^{d_{1} T}-1\right] \leq \delta(T)
\end{gathered}
$$

With the help of (47), (48), and (46) becomes $\dot{V}(t, Z(t)$, $Z(t-\tau)) \leq 0$. This implies immediately that all the solutions $z_{1}(t), \ldots, z_{n}(t)$ of the closed-loop system are globally bounded and well defined over $[0, \infty]$. This completes the proof of Theorem 7.

Remark 9. The controller is constructed in several steps. First, the control gains $K_{i}^{\prime} s$ are chosen as the coefficients of a Hurwitz polynomial. Then, based on the given growth condition of the nonlinearities and $c_{3}$, the scaling gain $L$ is chosen using the formula (24). Our last step is to find a proper sampling period $T$ according to inequalities (47) and (48).

Remark 10. The formulas (46) and (47) will yield a very small $T$ due to the conservative estimations used to simplify the proof. However, in practice we can actually use a relatively large sampling period for acceptable convergence performances. Moreover, with the recent technology advance, even a very small sampling period is no longer a hurdle in implementing the proposed sampled-data controller.

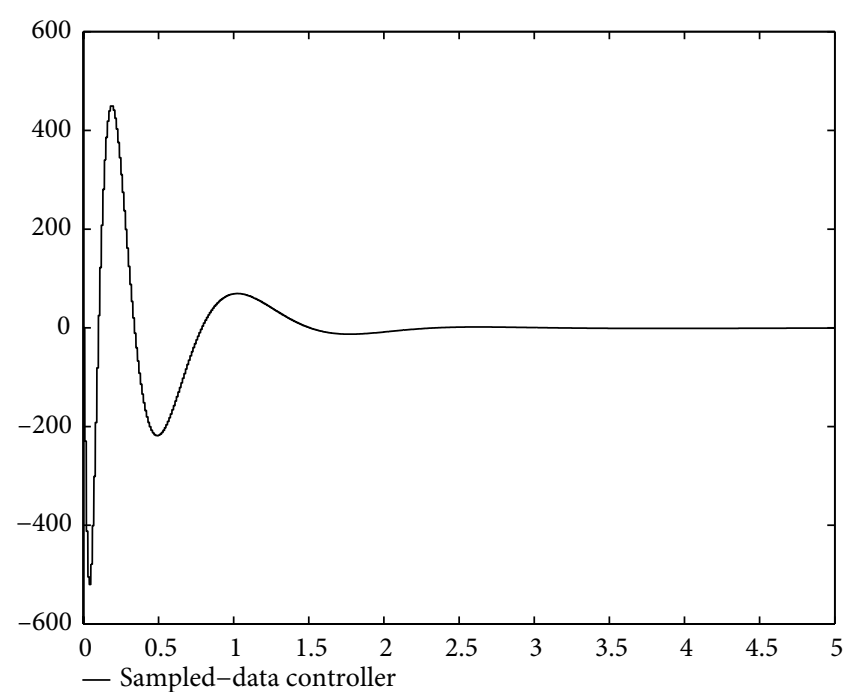

FIGURE 2: The sampled-data control signal transmitted through network.

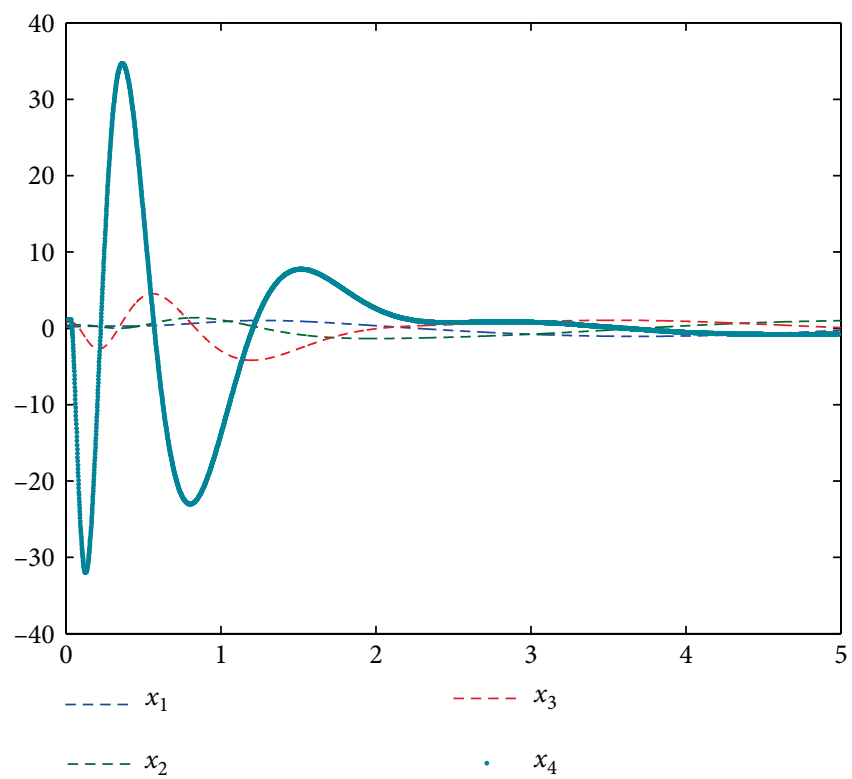

FIGURE 3: The response of states.

Remark 11. In this paper, the input delay and the system delay are both considered. Different from other transaction methods, we use the inductive proof method to get the criteria on $L$ and sampling period $T$ to make sure that the nonlinear system are globally stabilized. For a specific situation, we suppose the input delay and the system delay are the same. But it is not the necessary supposition. Our method can be easily applied to different types of delay combination.

Next, we use Theorem 7 to solve the single-link robot arm system while considering the transport delay via network.

\section{Simulation Results}

Example 12. Consider the following single-link robot arm system introduced in Qian and Lin [15] where the angular position $x_{2}$ is transported through network. 


$$
\begin{aligned}
& \dot{x}_{1}=x_{2}, \\
& \dot{x}_{2}=x_{3}-\frac{F_{2}(t)}{J_{2}} x_{2}(t-\tau)-\frac{k}{J_{2}} x_{1}-\frac{m g d}{J_{2}}\left(\cos x_{1}-1\right), \\
& \dot{x}_{3}=x_{4}, \\
& \dot{x}_{4}=u+\frac{k^{2}}{J_{1} J_{2} J_{3}^{2}} x_{1}-\frac{k}{J_{2} J_{3}} x_{3}-\frac{F_{1}(t)}{J_{1}} x_{4}, \\
& y=x_{1},
\end{aligned}
$$

where $x_{1}$ and $x_{3}$ denote the angular positions with respect to a fixed reference frame, of the link and respectively of the actuator shaft. $J_{1}, J_{2}, k, J_{3}, m, g, d$ are known parameters. $F_{1}(t)$ and $F_{2}(t)$ are viscous friction coefficients which are unknown but bounded by known constants. By the following relation

$$
\begin{aligned}
\left|\cos x_{1}-1\right| & \leq\left|x_{1}\right|, \\
\left|\frac{F_{2}(t)}{J_{2}} x_{2}(t-\tau)\right| & \leq c_{6}\left|x_{2}(t-\tau)\right|, \\
\left|\frac{F_{1}(t)}{J_{1}} x_{4}\right| & \leq c_{7}\left|x_{4}\right|, \quad \text { for constants } c_{6}, c_{7},
\end{aligned}
$$

it is clear that Assumption 1 holds for system (49).

Applying Theorem 7, where $\tau=T$, we can design the observer as

$$
\begin{aligned}
\widehat{z}((k+1) T)= & e^{L \hat{A} T} \hat{z}(k T) \\
& +\int_{0}^{T} e^{L \hat{A} s} d s[-L B K \hat{z}(k T-T)+L H C Z(k T)], \\
& k=0,1,2,3, \ldots,
\end{aligned}
$$

where

$$
\begin{aligned}
\hat{A} & =\left[\begin{array}{llll}
-3.43 & 1 & 0 & 0 \\
-3.92 & 0 & 1 & 0 \\
-1.86 & 0 & 0 & 1 \\
-0.31 & 0 & 0 & 0
\end{array}\right], \\
H & =\left[\begin{array}{l}
3.43 \\
3.92 \\
1.86 \\
0.31
\end{array}\right], \\
B & =\left[\begin{array}{l}
0 \\
0 \\
0 \\
1
\end{array}\right], \\
C & =\left[\begin{array}{llll}
1 & 0 & 0 & 0
\end{array}\right], \\
K & =\left[\begin{array}{llll}
0.31 & 1.86 & 3.92 & 3.43
\end{array}\right] .
\end{aligned}
$$

With the help of (14), the sampled-data controller can be designed as

$$
\begin{aligned}
u(t)= & L^{4} u((k-1) T)=-L^{4} k_{1} x_{1}((k-1) T) \\
& -L^{3} k_{2} x_{2}((k-1) T)-L^{2} k_{3} x_{3}((k-1) T)-L k_{4} x_{4}((k-1) T) .
\end{aligned}
$$

In the numerical simulation, $F_{1}(t)=0.1 \sin (t), F_{2}(t)=\cos (t)$, $J_{2}=10$ and the rest of parameters as 1 , the initial values $x(0)=\left[\begin{array}{llll}0.2 & 0.4 & 0.8 & 1\end{array}\right]^{T}$ and $\widehat{x}(0)=\left[\begin{array}{llll}0 & 0 & 0 & 0\end{array}\right]^{T}$. The detailed steps of the calculation of sampling period $T$ and $L$ are as follows:

(1) We select $L$ firstly according to the equation (24).

(2) Substitute $L$ into (47) and (48) to obtain the varying range of sampling period $T$.

(3) Select the most suitable $T$ to finish the simulation.

In the numerical simulation, $L$ is set to 6 and $T$ is set to $0.01 \mathrm{~s}$ in order to satisfy (47), (48) and (24) simultaneously. As shown in Figures 2 and 3, the sampled-data networked controller satisfies the global stabilization for the delay nonlinear system (49).

\section{Conclusion}

When the states are not measurable, we design a sampled-data output feedback controller to globally stabilize networked nonlinear time-delay systems. For the first time, we apply the inductive method to nonlinear networked control systems with one-sample-time input delay. The estimation technique is innovative and the simulation results show the effectiveness of our method. In the future, we will try to apply our theory to real systems, such as autonomous vehicle systems, aircraft systems, etc. We will also make further studies to extend our theory to switched systems and stochastic systems [28].

\section{Data Availability}

The data used to support the findings of this study are included in the supplementary material PDF file.

\section{Conflicts of Interest}

The authors declare that they have no conflicts of interest.

\section{Acknowledgments}

This research was supported by Natural Science Foundations of China (Grant nos. 61603194, 51706108) and Natural Science Fund for Colleges and Universities of Jiangsu Province (Grant nos. 16KJB120002, 17KJB510031).

\section{Supplementary Materials}

$\mathrm{M}$ files are simulation files which are used to simulate for Example 12 in our paper using Matlab Program. (Supplementary Materials)

\section{References}

[1] H. Chu, C. Qian, J. Yang, S. Xu, and W. Zha, "Global stabilization via sampled-data output feedback control for 
a class of nonlinear systems subject to time-delays," in 34th Chinese Control Conference, pp. 957-962, IEEE, Hangzhou, China, 2015.

[2] H. Chu, C. Qian, J. Yang, S. Xu, and Y. Liu, “ Almost disturbance decoupling for a class of nonlinear systems via sampled-data output feedback control," International Journal of Robust and Nonlinear Control, vol. 26, no. 10, pp. 2201-2215, 2016.

[3] B. A. Bamieh and J. Pearson Jr., "A general framework for linear periodic systems with applications to h/sup infinity/sampleddata control," IEEE Transactions on Automatic Control, vol. 37, no. 4, pp. 418-435, 1992.

[4] E. Fridman, A. Seuret, and J.-P. Richard, "Robust sampleddata stabilization of linear systems: an input delay approach," Automatica, vol. 40, no. 8, pp. 1441-1446, 2004.

[5] C. Byrnes and A. Isidori, "Steady state response, separation principle and the output regulation of nonlinear systems," in Proceedings of the 28th IEEE Conference on Decision and Control, pp. 2247-2251, IEEE, Tampa, FL, USA, 1989.

[6] H. Chu, C. Qian, R. Liu, and L. Di, "Global practical tracking of a class of nonlinear systems using linear sampled-data control," International Journal of Control, vol. 88, no. 9, pp. 1851-1860, 2015.

[7] R. F. Cunha, G. W. Gabriel, and J. C. Geromel, "Partial sampleddata state feedback control of markov jump linear systems," IFAC-PapersOnLine, vol. 51, no. 25, pp. 222-227, 2018.

[8] D. Nešić, A. Teel, and D. Carnevale, "Explicit computation of the sampling period in emulation of controllers for nonlinear sampled-data systems," IEEE Transactions on Automatic Control, vol. 54, no. 3, pp. 619-624, 2009.

[9] D. Nešić, A. R. Teel, and P. Kokotović, "Sufficient conditions for stabilization of sampled-data nonlinear systems via discretetime approximations," Systems \& Control Letters, vol. 38, no. 4, pp. 259-270, 1999.

[10] J. H. Ahrens, X. Tan, and H. K. Khalil, "Multirate sampleddata output feedback control with application to smart material actuated systems," IEEE Transactions on Automatic Control, vol. 54, no. 11, pp. 2518-2529, 2009.

[11] A. M. Dabroom and H. K. Khalil, "Output feedback sampleddata control of nonlinear systems using high-gain observers," IEEE Transactions on Automatic Control, vol. 46, no. 11, pp. 1712-1725, 2001.

[12] H. K. Khalil, "Performance recovery under output feedback sampled-data stabilization of a class of nonlinear systems," IEEE Transactions on Automatic Control, vol. 49, no. 12, pp. 2173-2184, 2004.

[13] H. Du, C. Qian, S. Li, and Z. Chu, "Global sampled-data output feedback stabilization for a class of uncertain nonlinear systems," Automatica, vol. 99, pp. 403-411, 2019.

[14] C. Qian and H. Du, "Global output feedback stabilization of a class of nonlinear systems via linear sampled-data control," IEEE Transactions on Automatic Control, vol. 57, no. 11, pp. 2934-2939, 2012.

[15] C. Qian and W. Lin, "Output feedback control of a class of nonlinear systems: a nonseparation principle paradigm," IEEE Transactions on Automatic Control, vol. 47, no. 10, pp. 17101715, 2002.

[16] W. Chen, J. Wu, and L. Jiao, "State-feedback stabilization for a class of stochastic time-delay nonlinear systems," International Journal of Robust and Nonlinear Control, vol. 22, no. 17, pp. 1921-1937, 2012.
[17] Z. Sun, Y. Shao, and C. Chen, "Fast finite-time stability and its application in adaptive control of high-order nonlinear system," Automatica, vol. 106, pp. 339-348, 2019.

[18] Z. Sun, M. Yun, and T. Li, "A new approach to fast global finitetime stabilization of high-order nonlinear system," Automatica, vol. 81, pp. 455-463, 2017.

[19] X. Zhang, L. Baron, Q. Liu, and E.-K. Boukas, "Design of Stabilizing Controllers With a Dynamic Gain for Feedforward Nonlinear Time-Delay Systems," IEEE Transactions on Automatic Control, vol. 56, no. 3, pp. 692-697, 2011.

[20] W. Ai, J. Zhai, and S. Fei, "Universal adaptive regulation for a class of nonlinear systems with unknown time delays and output function via output feedback," Journal of the Franklin Institute, vol. 350, no. 10, pp. 3168-3187, 2013.

[21] V. V. Assche, T. Ahmed-Ali, C. A. B. Hann, and F. LamnabhiLagarrigue, "High gain observer design for nonlinear systems with time varying delayed measurements," 18th IFAC Proceedings Volumes, vol. 44, no. 1, pp. 692-696, 2011.

[22] D. Zhang, Y. Shen, and X. Xia, "Continuous observer design for nonlinear systems with sampled and delayed output measurements," 19th IFAC Proceedings Volumes, vol. 47, no. 3, pp. 269-274, 2014.

[23] T. Ahmed-Ali, I. Karafyllis, and F. Lamnabhi-Lagarrigue, "Global exponential sampled-data observers for nonlinear systems with delayed measurements," IEEE Control Systems Letters, vol. 62, no. 7, pp. 539-549, 2013.

[24] T. Ahmed-Ali, V. V. Assche, J. Massieu, and P. Dorleans, "Continuous discrete observer for state affine systems with sampled and delayed measurements," IEEE Transactions on Automatic Control, vol. 58, no. 4, pp. 1085-1091, 2013.

[25] H. Du, C. Qian, Y. He, and Y. Cheng, "Global sampled-data output feedback stabilisation of a class of upper-triangular systems with input delay," IET Control Theory \& Applications, vol. 7, no. 10, pp. 1437-1446, 2013.

[26] X. Wang, H. Du, S. Li, and X. Zhou, "Global stabilization for a class of nonlinear systems with time delays via sampled-data output feedback," 20th IFAC World Congress, vol. 50, no. 1, pp. 16040-16045, 2017.

[27] W. Guan, "Adaptive output feedback control of a class of uncertain nonlinear systems with unknown time delays," International Journal of Systems Science, vol. 43, no. 4, pp. 682-690, 2012.

[28] C. Hua, G. Liu, L. Zhang, and X. Guan, “Cooperative stabilization for linear switched systems with asynchronous switching," IEEE Transactions on Systems Man Cybernetics-systems, vol. 49, no. 6, pp. 1081-1087, 2019. 


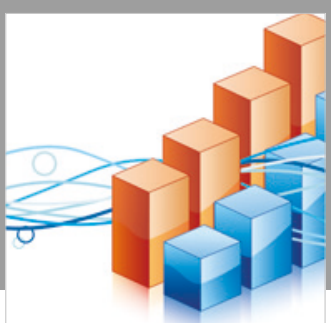

Advances in

Operations Research

\section{-n-m}
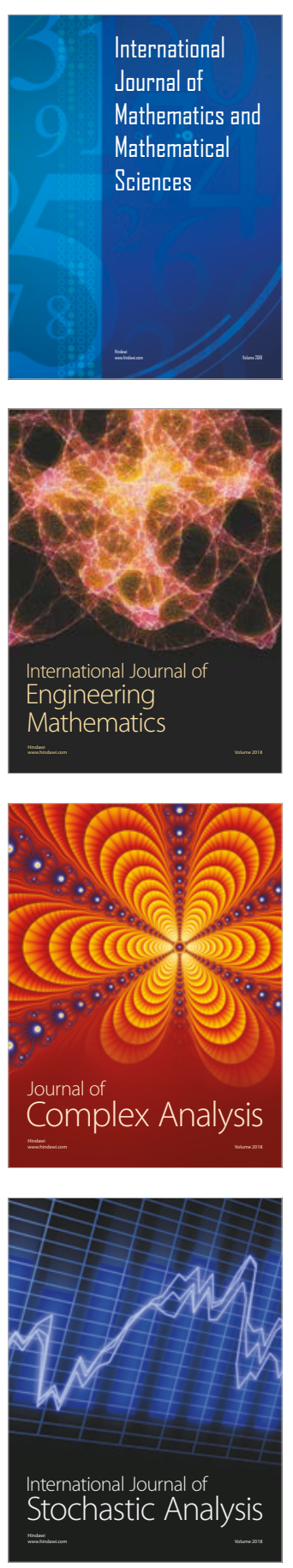
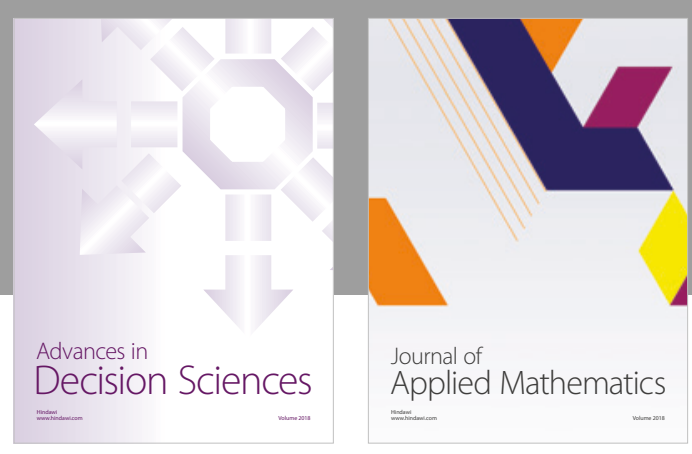

Journal of

Applied Mathematics
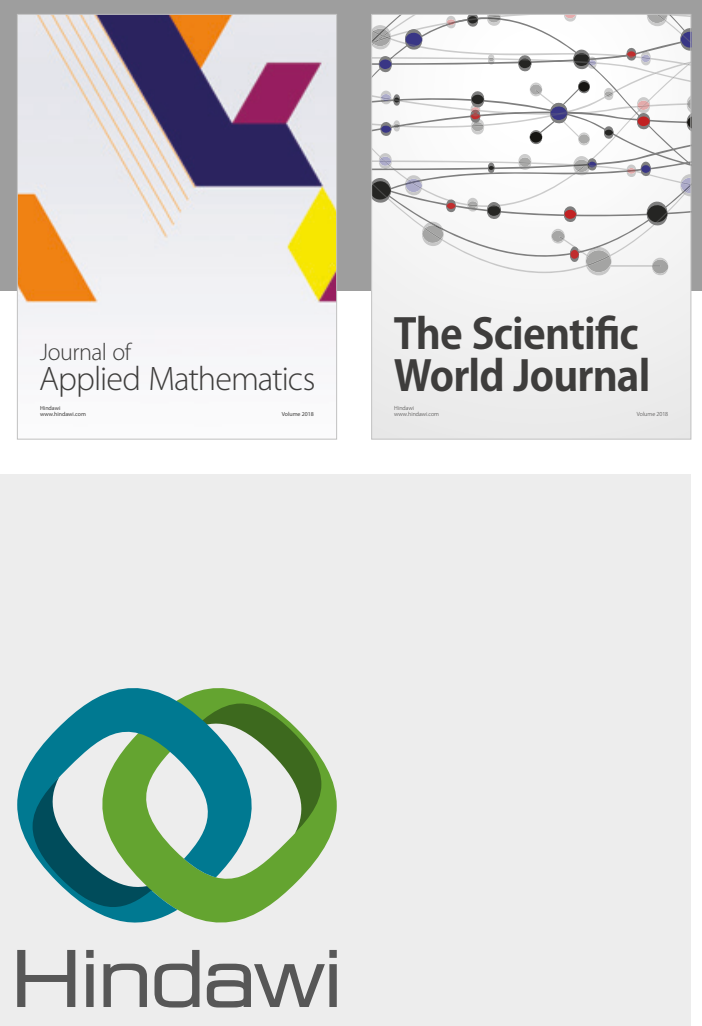

Submit your manuscripts at

www.hindawi.com

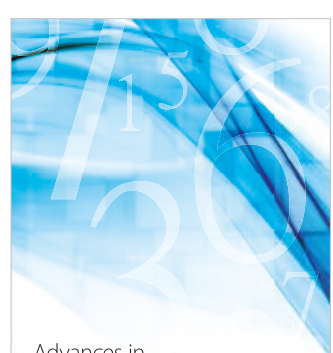

Advances in
Numerical Analysis
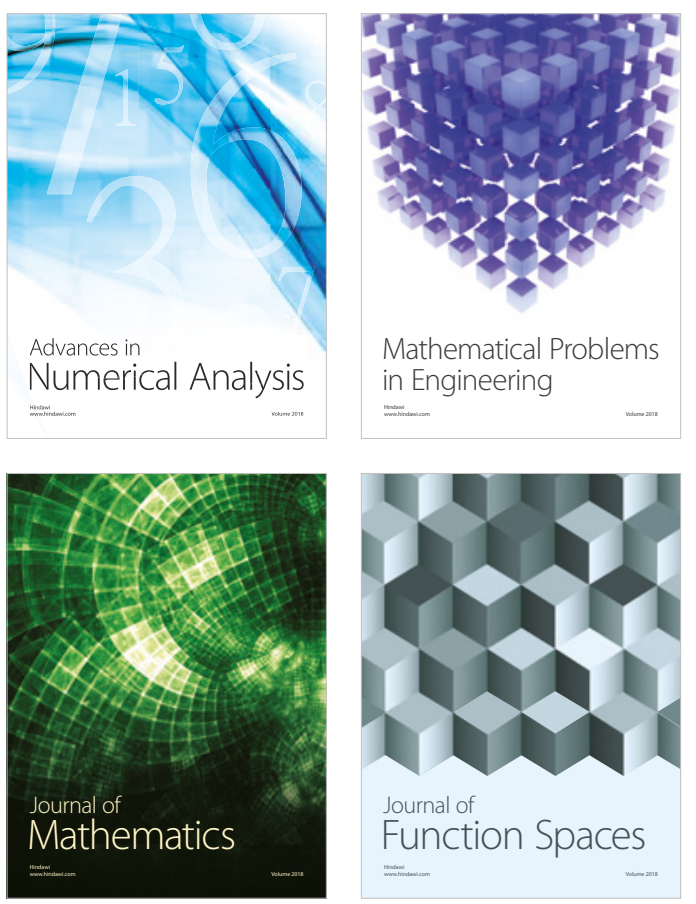

Mathematical Problems in Engineering

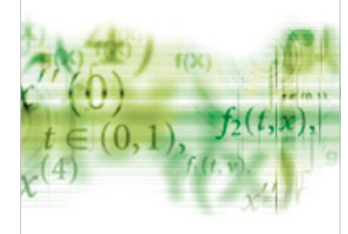

International Journal of

Differential Equations

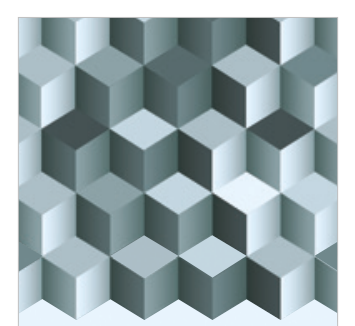

Journal of

Function Spaces
The Scientific

World Journal

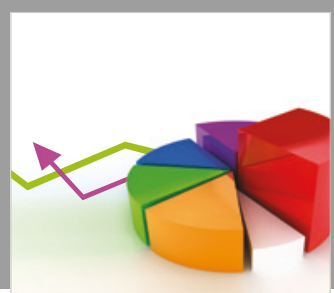

Journal of

Probability and Statistics
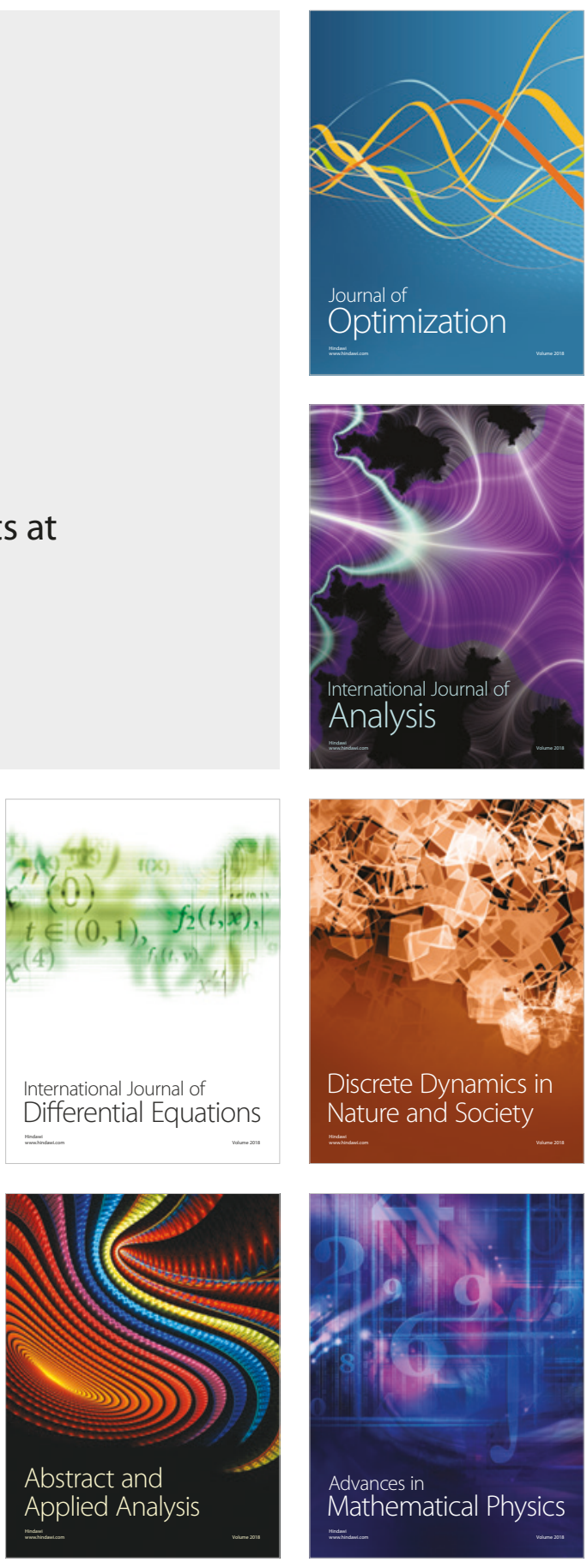